\title{
含电子给体乙炔二茂铁化合物的光谱及电化学性质
}

\author{
曹迁永 ${ }^{1, *} \quad$ 卢 金金 $^{1}$ 匡仁云 ${ }^{2}$ 李志华 1 杨震宇 ${ }^{1}$ \\ (1 南昌大学化学系, 南昌 330031; 2 井冈山大学化学化工学院, 江西吉安 343009)
}

\begin{abstract}
摘要： 合成了三种含电子给体乙炔基桥连二茂铁化合物 $\mathrm{Fc}-\mathrm{C} \equiv \mathrm{C}-\mathrm{Ph}-(p-\mathrm{OMe})(3 \mathrm{a}), \mathrm{Fc}-\mathrm{C} \equiv \mathrm{C}-\mathrm{Ph}-(p-$ $\left.\mathrm{NMe}_{2}\right)(3 \mathbf{b})$ 与 $\mathrm{Fc}-\mathrm{C} \equiv \mathrm{C}-\mathrm{Ph}-\left(p-\mathrm{NPh}_{2}\right)(3 \mathbf{c})$, 并对其结构、光化学及电化学性质进行了表征. 吸收光谱表明, 所有 化合物在可见光区 400-550 nm 都可归属为 $\mathrm{Fe}(\mathrm{II}) \rightarrow \mathrm{Cp}-\mathrm{C} \equiv \mathrm{C}-\mathrm{Ph}-(p-\mathrm{R})(\mathrm{Cp}$ 为环戊二烯基)的金属到配体的 电荷跃迁(MLCT) 光谱, 同时检测到 3a 与 3c 氧化态在近红外区 (分别位于 946 与 $1044 \mathrm{~nm}$ ) 可归属为 $\mathrm{Cp}-\mathrm{C} \equiv$ $\mathrm{C}-\mathrm{Ph}-(p-\mathrm{R}) \rightarrow \mathrm{Fe}(\mathrm{III})$ 的配体到金属的电荷跃迁 (LMCT). 电化学测试结果表明, 三种化合物都有稳定的 $\mathrm{Fc}^{+} / \mathrm{Fc}$ 可逆氧化还原电位, 且 $3 b$ 与 $3 c$ 还出现 $\mathrm{Ph}-\mathrm{NR}_{2}$ 的氧化电位. $3 b$ 对质子表现出光及电化学敏感性, 随着质子的 加人, 其 MLCT 光谱红移, $\mathrm{Fc}^{+} / \mathrm{Fc}$ 电位正移, 而 $\mathrm{Ph}-\mathrm{NR}_{2}^{+} / \mathrm{Ph}-\mathrm{NR}_{2}$ 峰消失.
\end{abstract}

关键词：二茂铁; 质子化; 吸收光谱; 氧化; 电化学

中图分类号: O646; O644

\section{Optical and Electrochemical Properties of Ethynyl-Bridged Ferrocenes with Electron Donor Groups}

\author{
CAO Qian-Yong ${ }^{1, *} \quad$ LU Xin $^{1} \quad$ KUANG Ren-Yun ${ }^{2} \quad$ LI Zhi-Hua ${ }^{1} \quad$ YANG Zhen-Yu ${ }^{1}$ \\ ( ${ }^{1}$ Department of Chemistry, Nanchang University, Nanchang 330031, P. R. China; \\ ${ }^{2}$ College of Chemistry and Chemical Engineering, Jinggangshan University, Ji'an 343009, Jiangxi Province, P. R. China)
}

\begin{abstract}
We present the synthesis, optical and electrochemical properties of three ethynyl-bridged ferrocenes with electron donor groups, $\mathrm{Fc}-\mathrm{C} \equiv \mathrm{C}-\mathrm{Ph}-(p-\mathrm{OMe})(3 \mathbf{a}), \mathrm{Fc}-\mathrm{C} \equiv \mathrm{C}-\mathrm{Ph}-\left(p-\mathrm{NMe}_{2}\right)(3 \mathbf{b})$ and $\mathrm{Fc}-\mathrm{C} \equiv \mathrm{C}-\mathrm{Ph}-\left(p-\mathrm{NPh}_{2}\right)(3 \mathbf{c})$. All three compounds show a $\mathrm{Fe}(\mathrm{II}) \rightarrow \mathrm{Cp}-\mathrm{C} \equiv \mathrm{C}-\mathrm{Ph}-(p-\mathrm{R})(\mathrm{Cp}=$ cyclopentadienyl) metal to ligand charge transition (MLCT) in 400-550 nm. Upon oxidation, 3a and 3c show a $\mathrm{Cp}-\mathrm{C} \equiv \mathrm{C}-\mathrm{Ph}-(p-\mathrm{R}) \rightarrow \mathrm{Fe}$ (III) ligand to metal charge transition (LMCT) in the near-IR range (946 and $1044 \mathrm{~nm})$. A reversible $\mathrm{Fc}^{+} / \mathrm{Fc}$ potential for $\mathbf{3 a}-\mathbf{3 c}$ and an irreversible $\mathrm{Ph}-\mathrm{NR}_{2}^{+} / \mathrm{Ph}-\mathrm{NR}_{2}$ potential for $\mathbf{3 b}$ and $\mathbf{3 c}$ are observed by in the cyclic and differential pulse voltammetry. Finally, 3b shows an optical and electrochemical response upon protonation, with a red shift of the MLCT transition, an anodic shift of the $\mathrm{Fc}^{+} / \mathrm{Fc}$ potential, and disappearance of the $\mathrm{Ph}-\mathrm{NR}_{2}^{+} / \mathrm{Ph}-\mathrm{NR}_{2}$ peak.
\end{abstract}

Key Words: Ferrocene; Protonation; Absorption spectrum; Oxidation; Electrochemistry

含有延伸 $\pi$ 电子共轭体系的金属有机化合物由 于其在光、电、磁性等方面所具有的独特性能, 已经 引起了人们的广泛重视和深人研究 ${ }^{[1-3]}$. 二茂铁及其 衍生物由于好的芳香性、氧化还原活性、稳定性及易
化学修饰等特点, 在这一领域中具有重要的地位 ${ }^{[4]}$. 近年来, 这类共轭体系二茂铁化合物在非线性光化 学、化学传感器、超分子组装及异核金属有机化合物 合成等方面的研究获得了较大的进展 ${ }^{[5-10]}$. 在这些共

\footnotetext{
Received: January 5, 2010; Revised: April 6, 2010; Published on Web: June 25, 2010.

"Corresponding author. Email: cqyong@ncu.edu.cn; Tel: +86-791-3969252.

The project was supported by the National Natural Science Foundation of China (20963007), Bureau of Education of Jiangxi Province, China (GJJ09074) and Program for Innovative Research Team of Nanchang University, China.

国家自然科学基金(20963007), 江西省教育厅自然科学基金(GJJ09074)和南昌大学创新团队基金资助

(C) Editorial office of Acta Physico-Chimica Sinica
} 
轭二茂铁化合物中, 二茂铁部分 $(\mathrm{Fc})$ 一般作为电子 给体(D), 而其余部分常含吸电子基作为电子受体 (A), 通过双键或三键等不饱和键相连形成 $\mathrm{D}(\mathrm{Fc})-\pi$ $\mathrm{A}$ 二元化合物, 给体与受体部分通过不饱和键有效 地进行分子内电荷转移. 而相对于其余部分含供 电子基的 $\mathrm{D}(\mathrm{Fc})-\pi-\mathrm{D}$ 二元化合物的研究则相对较 少 ${ }^{[11-13]}$. 由于二茂铁稳定的氧化态, 通过适当的化学 氧化或电化学氧化, 这种 $\mathrm{D}(\mathrm{Fc})-\pi-\mathrm{D}$ 二元化合物可 变为 $\mathrm{A}\left(\mathrm{Fc}^{+}\right)-\pi-\mathrm{D}$, 有望应用于分子导线及近红外光 检测等领域 ${ }^{[5]}$. 在此, 我们合成了三种 $\mathrm{D}(\mathrm{Fc})-\pi-\mathrm{D}$ 型二 元化合物, $\mathrm{Fc}-\mathrm{C} \equiv \mathrm{C}-\mathrm{Ph}-(p-\mathrm{OMe})(3 \mathrm{a}), \mathrm{Fc}-\mathrm{C} \equiv$ $\mathrm{C}-\mathrm{Ph}-\left(p-\mathrm{NMe}_{2}\right)(3 \mathbf{b})$ 与 $\mathrm{Fc}-\mathrm{C} \equiv \mathrm{C}-\mathrm{Ph}-\left(p-\mathrm{NPh}_{2}\right)$ (3c). 研究了它们中性及氧化态电子吸收光谱及电 化学性质, 并对化合物 $3 b$ 进行了质子识别研究.

\section{1 实验部分}

\section{1 试剂、仪器及相关测试}

乙炔二茂铁 (2) 与 $N, N$-二苯基-对溴苯胺 (1c) 按 文献方法 ${ }^{[14-15]}$ 合成, $\mathrm{Pd}\left(\mathrm{PPh}_{3}\right)_{2} \mathrm{Cl}_{2}$ 及 $\mathrm{HBF}_{4} \cdot \mathrm{Et}_{2} \mathrm{O}$ (分析 纯)从英国 Alfa Aeser 公司购买, 其它试剂及药品(分 析纯)购于国药集团化学试剂公司. 无水溶剂按文献 方法纯化 ${ }^{[16]}$.

使用日本 Shimadzu UV2300 型紫外光谱仪; 美 国 PE 公司 Spectrum One 傅里叶转换红外仪 $(\mathrm{KBr}$ 压片); 美国 Varian $400 \mathrm{MHz}$ 核磁共振仪, 四甲基硅 烷 (TMS) 为内标; 上海辰华电化学工作站 CHI $610 \mathrm{C}$; 德国 IKA-RET 磁力搅拌器.

电化学相关测试在上海辰华仪器有限公司生产 的 CHI $610 \mathrm{C}$ 电化学工作站上进行, 测试时采用三 电极体系, 以铂圆盘电极为工作电极、铂丝电极为对 电极、 $\mathrm{Ag} / \mathrm{Ag}^{+}$为参比电极 $\left(\mathrm{Ag}^{+}\right.$的浓度 $\left.0.10 \mathrm{~mol} \cdot \mathrm{L}^{-1}\right)$, 六氟磷酸四丁基铵 $\left(0.10 \mathrm{~mol} \cdot \mathrm{L}^{-1}\right)$ 为支持电解质, 乙 腈为溶剂. 测试样品浓度为 $500 \mu \mathrm{mol} \cdot \mathrm{L}^{-1}$, 测定之前 通 $\mathrm{N}_{2}, 15-20 \mathrm{~min}$ 除氧, 在氮气气氛中进行测定, 利 用循环伏安法 $(\mathrm{CV})$ 和常规差分脉冲伏安法(DPV)(脉 冲宽度为 $50 \mathrm{~ms}$ ) 进行测定, 其扫描范围为 $-0.100-$ $1.000 \mathrm{~V}$, 扫描速率为 $0.05 \mathrm{~V} \cdot \mathrm{s}^{-1}$.

\section{2 合 成}

化合物 3a-3c 由 2 与溴代苯在 $\mathrm{Pd}\left(\mathrm{PPh}_{3}\right)_{2} \mathrm{Cl}_{2} / \mathrm{CuI}$ 催化下, 通过 Sonogashira 偶联反应 ${ }^{[17-181}$ 得到(见图1). 其基本反应步骤如下: 在 $\mathrm{N}_{2}$ 保护下, 将 $2 \mathrm{mmol}(420$ $\mathrm{mg}) \boldsymbol{2}$ 和 $2 \mathrm{mmol}$ 溴代苯 $(\mathbf{1}), 0.06 \mathrm{mmol} \mathrm{Pd}\left(\mathrm{PPh}_{3}\right)_{2} \mathrm{Cl}_{2}$, $0.06 \mathrm{mmol} \mathrm{CuI}$ 加人 $40 \mathrm{~mL}$ 的无水三乙胺和四氢呋

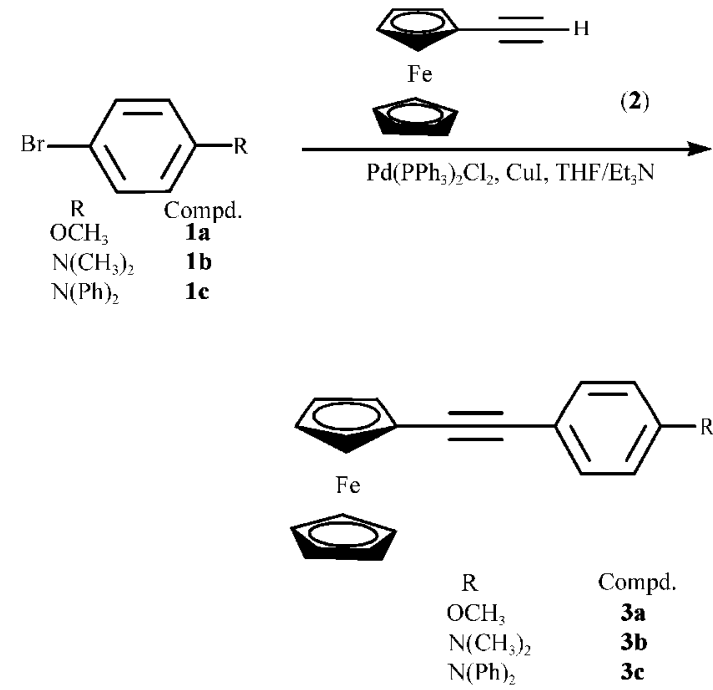

图 $13 a-3 c$ 的合成方法

Fig.1 Synthetic approach for $3 a-3 c$

喃(体积比为 $1 / 1$ )中, 搅拌回流 $18 \mathrm{~h}$. 反应结束后, 抽 滤, 将滤液旋干, 粗产品通过硅胶柱分离 (洗脱剂: 乙酸乙酯/石油醚)得到黄色固体.

3a: $379 \mathrm{mg}$, 产率 $60 \%$. ${ }^{1} \mathrm{H}$ NMR $\left(400 \mathrm{MHz}, \mathrm{CDCl}_{3}\right.$, $25{ }^{\circ} \mathrm{C}$, TMS) $\delta$ : 3.18(s, 3H), 3.93-3.96 (d, 2H), 4.11 (s, $5 \mathrm{H}), 4.48-4.51(\mathrm{~d}, 2 \mathrm{H}), 6.57-6.62(\mathrm{~d}, 2 \mathrm{H}), 7.47-7.52$ $(\mathrm{m}, 2 \mathrm{H})$. 分析计算得 $\mathrm{C}_{19} \mathrm{H}_{10} \mathrm{FeO}$ (316.06): C, 72.18\%; $\mathrm{H}, 5.10 \%$; O, $5.06 \%$. 实验得到: C, 72.33\%; H, 5.01\%; O, $5.16 \%$. IR $\left(\mathrm{KBr}, \mathrm{cm}^{-1}\right)$ : 1258, 1389, 2180.

3b: $467 \mathrm{mg}$, 产率 71\%. ${ }^{1} \mathrm{H} \mathrm{NMR} \quad(400 \mathrm{MHz}$, $\mathrm{CDCl}_{3}, 25{ }^{\circ} \mathrm{C}$, TMS $) \delta: 2.98(\mathrm{~s}, 6 \mathrm{H}), 4.19-4.2(\mathrm{~d}, 2 \mathrm{H})$, $4.23(\mathrm{~s}, 5 \mathrm{H}), 4.46-4.47$ (d, 2H), 6.63-6.66 (d, 2H), 7.35$7.38(\mathrm{~d}, 2 \mathrm{H})$. 分析计算得 $\mathrm{C}_{20} \mathrm{H}_{19} \mathrm{FeN}$ (329.09): $\mathrm{C}$, $72.97 \%$; H, $5.82 \%$; N , 4.25\%. 实验得到: C, $72.85 \%$; $\mathrm{H}, 5.90 \%$; N, 4.14\%. IR (KBr, cm $\left.{ }^{-1}\right): 1378,2215$.

3c: $607 \mathrm{mg}$, 产率67\%. ${ }^{1} \mathrm{H}$ NMR $(400 \mathrm{MHz}$, $\mathrm{CDCl}_{3}, 25{ }^{\circ} \mathrm{C}$, TMS) $\delta: 4.22-4.23(\mathrm{~m}, 2 \mathrm{H}), 4.26(\mathrm{~s}, 5 \mathrm{H})$, 4.47-4.48 (t, 2H), 6.98-7.0 (d, 2H), 7.03-7.05 (d, 2H), $7.06-7.12(\mathrm{~m}, 4 \mathrm{H}), 7.27-6.29(\mathrm{~d}, 4 \mathrm{H}), 7.32-7.34(\mathrm{~d}$, $2 \mathrm{H})$. 分析计算得到 $\mathrm{C}_{30} \mathrm{H}_{23} \mathrm{FeN}$ (453.12) : C, 79.48\%; $\mathrm{H}$, $5.11 \%$; N $3.09 \%$. 实验得到: C, 79.30\%; H, 5.23\%; N, $3.02 \%$. IR $\left(\mathrm{KBr}, \mathrm{cm}^{-1}\right): 2210$.

\section{2 结果与讨论}

\section{1 化合物的吸收光谱及电化学性质}

化合物 3a-3c 在乙腈溶液中的吸收光谱见图 2 , 详细的光谱数据见表 1 . 从表 1 和图 2 可看出, 三 


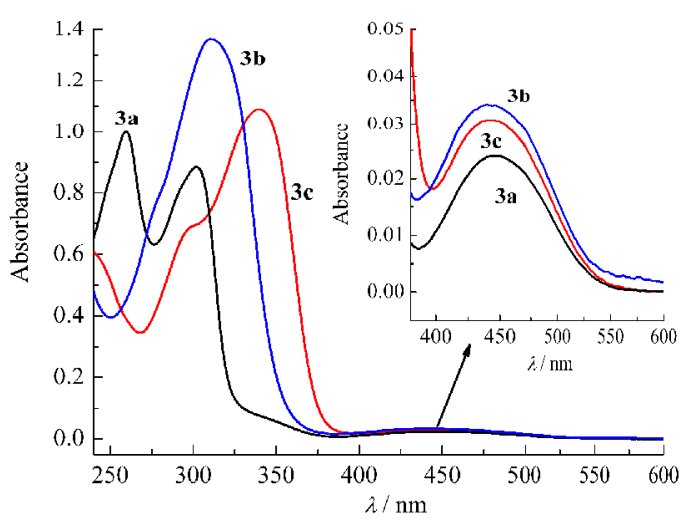

图 $23 \mathrm{a}-3 \mathrm{c}\left(30 \mu \mathrm{mol} \cdot \mathrm{L}^{-1}\right)$ 在 $\mathrm{CH}_{3} \mathrm{CN}$ 溶液中的 紫外-可见吸收光谱

Fig.2 UV-Vis spectra of $3 a-3 c\left(30 \mu \mathrm{mol} \cdot \mathrm{L}^{-1}\right)$ in $\mathrm{CH}_{3} \mathrm{CN}$ solution

inset: enlarge figure in the range of $380-600 \mathrm{~nm}$

种化合物在紫外区都有强的吸收 $(\lambda<380 \mathrm{~nm})$, 可归 属为配体 $\mathrm{Cp}-\mathrm{C} \equiv \mathrm{C}-\mathrm{Ph}-(p-\mathrm{R})(\mathrm{Cp}$ 为环戊二烯 基)内部 $\pi-\pi^{*}$ 或 $n-\pi *$ 跃迁. 同时, 在可见光区 400$550 \mathrm{~nm}$ 处有一低能吸收, 吸收峰分别位于 446、440 和 $443 \mathrm{~nm}$, 可以归属为 $\mathrm{Fe}(\mathrm{II}) \rightarrow \mathrm{Cp}-\mathrm{C} \equiv \mathrm{C}-\mathrm{Ph}-(p-$ $\mathrm{R})$ 的 MLCT 带 ${ }^{[4-5]}$. 该吸收峰相对于含吸电子基 - $\mathrm{NO}_{2}$ 的 D-A 二元化合物有一定的蓝移 ${ }^{[19]}$, 这与文 献报道结果一致, 即吸电子基使 MLCT 光谱红移, 而供电子基导致蓝移.

通过循环伏安 $(\mathrm{CV})$ 和常规差分脉冲伏安(DPV) 法表征了所有化合物的的电化学性质(表 1 和图 3). 对于 3a, 在循环伏安曲线中, 半波电位 $E_{1 / 2}=0.193 \mathrm{~V}$ $(\Delta E=0.088 \mathrm{~V})$ 处出现一个可归属为 $\mathrm{Fc}^{+} / \mathrm{Fc}$ 的单电子 可逆氧化还原峰. 对于 $3 \mathbf{b}$ 和 $3 \mathbf{c}$, 除观察到 $E_{1 / 2}$ 分别 位于 0.161 及 $0.187 \mathrm{~V}$, 归属为 $\mathrm{Fc}^{+} / \mathrm{Fc}$ 的单电子可逆 氧化还原峰外, 同时在阳极电位峰 $E_{\mathrm{pa}}$ 为 0.632 和 $0.788 \mathrm{~V}$ 处出现单电子非可逆氧化还原峰, 可归属为 苯胺 $\mathrm{N}$ 原子 $\mathrm{Ph}-\mathrm{NR}_{2}^{+} / \mathrm{Ph}-\mathrm{NR}_{2}$ 电位, 同样的结果在 文献中也有报道 ${ }^{[20]}$. 与 $\mathrm{CV}$ 法相一致, 在 DPV 法中, $3 \mathbf{a}$ 仅在 $0.179 \mathrm{~V}$ 处出现单氧化峰, 而 $3 \mathbf{b}$ 和 $3 \mathbf{c}$ 都出
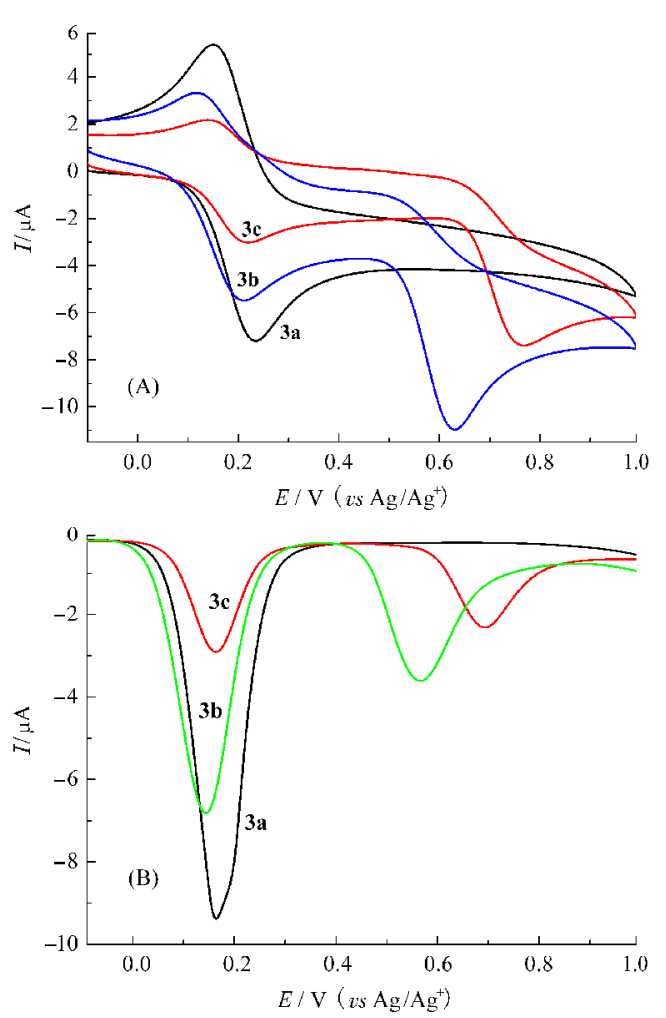

图 $33 \mathrm{a}-3 \mathrm{c}\left(500 \mu \mathrm{mol} \cdot \mathrm{L}^{-1}\right)$ 在 $\mathrm{CH}_{3} \mathrm{CN}$ 溶液中的循环 伏安谱图(A)和在 $\mathrm{CH}_{3} \mathrm{CN}$ 溶液中常规差分脉冲谱图 $(50 \mathrm{~ms}$ 脉冲宽度 $)(B)$

Fig.3 CVs of $3 a-3 c\left(500 \mu \mathrm{mol} \cdot \mathrm{L}^{-1}\right)$ in $\mathrm{CH}_{3} \mathrm{CN}$ solution (A) and DPVs (50 ms pulse width) of $3 a-3 c$ $\left(500 \mu \mathrm{mol} \cdot \mathrm{L}^{-1}\right)$ in $\mathrm{CH}_{3} \mathrm{CN}$ solution $(\mathrm{B})$

现了两个氧化峰, 分别对应为 $\mathrm{Fc}(3 \mathrm{~b}$ 的 $0.144 \mathrm{~V}$ 和 $3 \mathrm{c}$ 的 $0.162 \mathrm{~V})$ 及苯胺 $\mathrm{N}$ 原子 $(3 \mathrm{~b} 0.564 \mathrm{~V}$ 和 $3 \mathrm{c} 0.692$ $\mathrm{V})$ 的得失电子过程. 同时, 通过比较 $\mathrm{Fc}^{+} / \mathrm{Fc}$ 电位, 发 现其电位按 $3 a 、 3 c 、 3 b$ 的顺序减少, 且都小于含吸电 子取代基化合物 $\mathrm{Fc}-\mathrm{C} \equiv \mathrm{C}-\mathrm{Ph}-\left(p-\mathrm{NO}_{2}\right)$ 氧化还原 电位 $(0.20 \mathrm{~V} \text {, 相对于二茂铁电位 })^{[12]}$. 这与取代基的 给电子能力相一致: 吸电子取代基使 $\mathrm{Fc}^{+} / \mathrm{Fc}$ 电位增 大, 而供电子取代基使 $\mathrm{Fc}^{+} / \mathrm{Fc}$ 电位减少. $-\mathrm{OCH}_{3}$ 、 - $\mathrm{NPh}_{2}$ 、一 $\mathrm{NMe}_{2}$ 都为供电子取代基, 且供电子能力 依次增强.

表 $13 a-3 c$ 的紫外-可见-近红外吸收数据和电化学数据

Table 1 UV-Vis-NIR absorption and electrochemical data of 3a-3c

\begin{tabular}{|c|c|c|c|c|c|c|}
\hline & \multicolumn{2}{|r|}{$\lambda_{\max } / \mathrm{nm}\left(10^{-3} \varepsilon /\left(\mathrm{L} \cdot \mathrm{mol}^{-1} \cdot \mathrm{cm}^{-1}\right)\right)^{\mathrm{a}}$} & \multirow{2}{*}{$E_{1 / 2}(\Delta E)^{\mathrm{d}}$} & \multirow{2}{*}{$E_{\mathrm{pa}}^{\mathrm{d}}$} & \multirow{2}{*}{$E_{\mathrm{pl}}^{\mathrm{e}}$} & \multirow{2}{*}{$E_{\mathrm{p} 2}^{\mathrm{e}}$} \\
\hline & Neutral & Oxidized $^{\mathrm{b}}$ & & & & \\
\hline $3 a$ & $302(29.5), 446(0.8)$ & 292 (19.7), 409 (6.5), 491 (5.5), 946 (1.0) & $0.193(0.088)$ & - & 0.179 & - \\
\hline $3 c$ & 340 (36.3), 443 (1.0) & 295 (27.3), 327 (25.5), 493 (6.4), 1044 (1.4) & $0.187(0.084)$ & 0.788 & 0.162 & 0.692 \\
\hline
\end{tabular}

a by UV-Vis-NIR, high-energy bands $(>290 \mathrm{~nm})$ not given; ${ }^{\mathrm{b}}$ after oxidized by added $60 \mu \mathrm{mol} \cdot \mathrm{L}^{-1} \mathrm{Cu}(\mathrm{OTf})_{2}$; ${ }^{\mathrm{c}} \mathrm{Shoulder}$,

${ }^{\mathrm{d}}$ cyclic voltammetry $(\mathrm{CV}), E_{1 / 2}$ and $\Delta E$ (given in parentheses) $\left(v s \mathrm{Ag} / \mathrm{Ag}^{+}\right)$, the $E_{1 / 2}$ of unsubstituted ferrocene is $0.037 \mathrm{~V}\left(v s \mathrm{Ag} / \mathrm{Ag}^{+}\right)$, ${ }^{\mathrm{e}}$ differential pulse voltammetry (DPV), $E_{\mathrm{p}}\left(v s \mathrm{Ag} / \mathrm{Ag}^{+}\right), 50 \mathrm{~ms}$ pulse width; $E_{1 / 2}$ : half potential; $\varepsilon$ : molar absorbance coefficient; $E_{\mathrm{pa}}$ : anodic potential peak; $E_{\mathrm{p} 1}:$ the first oxidation potential peak; $E_{\mathrm{p} 2}$ : the second oxidation potential peak 


\section{2 化合物的化学氧化}

二茂铁化合物具有稳定的氧化态, 通过适当的 电化学氧化或化学氧化剂氧化, 可将 $\mathrm{Fc} \rightarrow \mathrm{Fc}^{+}$, 这在 研究化合物分子内电荷转移机理及分子导线设计等 领域有重要的意义 ${ }^{[21]}$. 在此我们以三氟甲基磺酸铜 作为氧化剂 ${ }^{[22]}$, 研究了 $3 \mathrm{a}-3 \mathrm{c}$ 氧化态 $\mathrm{Fc}^{+}-\mathrm{C} \equiv \mathrm{C}-$ $\mathrm{Ph}$ - $(p-\mathrm{R})$ 在 200-1100 nm 处的电子光谱变化. 其氧 化态时的电子光谱数据见表 1 , 同时图 4 给出了 $3 \mathrm{c}$ 不断滴加氧化剂时光谱变化. 从图 4 中可看出, 随着 氧化剂浓度的增加, $3 \mathrm{c}$ 在紫外光区 $340 \mathrm{~nm}$ 吸收峰 减弱并蓝移至 $327 \mathrm{~nm}, 300 \mathrm{~nm}$ 吸收峰增强蓝移至 $295 \mathrm{~nm}$; 在可见光区 410 及 $493 \mathrm{~nm}$ 出现两个新的 宽的吸收峰. 同时, 在近红外区 $\lambda_{\text {max }}=1044 \mathrm{~nm}$ (摩尔 吸光系数 $\left.\varepsilon=1.4 \times 10^{3} \mathrm{~L} \cdot \mathrm{mol}^{-1} \cdot \mathrm{cm}^{-1}\right)$ 出现了一个宽的 可归属为 $\mathrm{Cp}-\mathrm{C} \equiv \mathrm{C}-\mathrm{Ph}-(p-\mathrm{R}) \rightarrow \mathrm{Fe}$ (III) 的 LMCT 光谱 ${ }^{[11,23]}$. 在 311 和 $365 \mathrm{~nm}$ 处的等吸收点表明两化 学氧化过程中仅出现 $3 c$ 与氧化态 $3 c^{+}$两种组份. $3 \mathbf{a}^{+}$ 的电子光谱同 $3 c$ 类似, 其 LMCT 光谱 $\lambda_{\text {max }}=946 \mathrm{~nm}$ $\left(\varepsilon=1.0 \times 10^{3} \mathrm{~L} \cdot \mathrm{mol}^{-1} \cdot \mathrm{cm}^{-1}\right)$. 而对于 $3 \mathbf{b}$, 滴加氧化剂时 在 UV-Vis 区域光谱变化类似于 3a 与 $\mathbf{3} \mathbf{c}$, 其在紫外 区 $310 \mathrm{~nm}$ 处吸收峰减弱直至消失, 伴随 $352 \mathrm{~nm}$ 出 现新的吸收峰 $\left(\varepsilon=25.6 \times 10^{3} \mathrm{~L} \cdot \mathrm{mol}^{-1} \cdot \mathrm{cm}^{-1}\right)$; 在可见光 区 410-650 nm 出现宽的低能吸收. 同时, 在 274 和 $339 \mathrm{~nm}$ 处出现两个等吸收点. 在仪器测试范围 200-1100 nm, 未检测到近红外区吸收, 这可能因为 $3 \mathbf{b}^{+}$的 LMCT 光谱处于更低级, 因为一 $\mathrm{NMe}_{2}$ 相比一 $\mathrm{NPh}_{2}$ 与一 $\mathrm{OCH}_{3}$ 为更强的供电子基. 这一点同样可 从 $3 c$ 与 $3 a$ 及文献中报道不含吸电子基 $\mathrm{Fc}-\mathrm{C} \equiv$

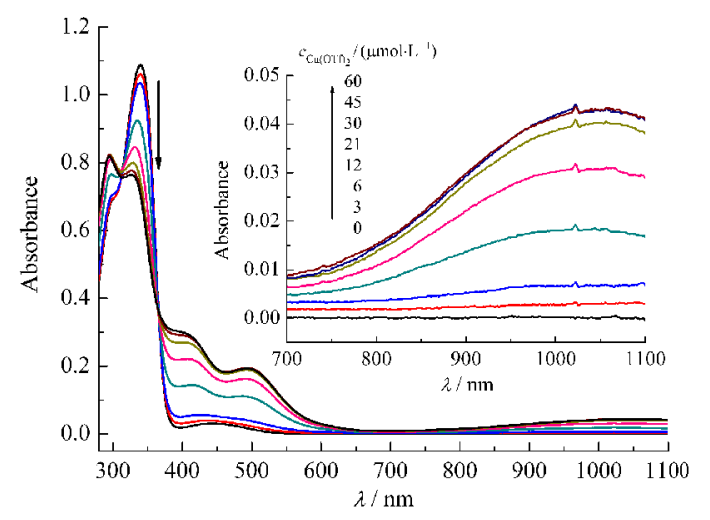

图 $43 \mathrm{c}\left(30 \mu \mathrm{mol} \cdot \mathrm{L}^{-1}\right)$ 在乙腈中滴定 $\mathrm{Cu}(\mathrm{OTf})_{2}$ (0-60 $\left.\mu \mathrm{mol} \cdot \mathrm{L}^{-1}\right)$ 的紫外-可见-近红外光谱图

Fig.4 UV-Vis-NIR spectra of $3 c\left(30 \mu \mathrm{mol} \cdot \mathrm{L}^{-1}\right)$ on titration with $\mathrm{Cu}(\mathrm{OTf})_{2}\left(0-60 \mu \mathrm{mol} \cdot \mathrm{L}^{-1}\right)$ in $\mathrm{CH}_{3} \mathrm{CN}$ solution

inset: enlarge figure in the range of 700-1100 nm
$\mathrm{C}$ - $\mathrm{Ph}$ 的吸收光谱得到证实 ${ }^{[11]}$. 随着供电子能力减 弱, 其 LMCT 最大吸收峰分别位于 1044、946 及 797 $\mathrm{nm}$ 处.

\section{3 化合物 $3 b$ 的质子识别}

由于 $3 b$ 中的 $N$ 原子含有孤对电子, 具有一定 的碱性. 通过质子化, 一 $\mathrm{NMe}_{2}$ 由供电子基转变为吸 电子基一 $\mathrm{NHMe}_{2}^{+}$, 从而使化合物的电子谱及电化学 性质发生改变. 基于这一原理, 质子识别的光化学及 电化学传感器已有文献报道 ${ }^{[24-25]}$. 在此, 我们研究了 化合物 $\mathbf{3 b}$ 滴加不同浓度质子时的电子光谱及电化 学性质的变化.

$3 \mathbf{b}$ 在乙腈溶液中滴加 $\mathrm{HBF}_{4} \cdot \mathrm{Et}_{2} \mathrm{O}$ 的吸收光谱 变化见图 5. 从图中可看出, 随着 $\mathrm{HBF}_{4} \cdot \mathrm{Et}_{2} \mathrm{O}$ 浓度的 增加, 在紫外光区 $310 \mathrm{~nm}$ 吸收峰减弱并蓝移到 306 $\mathrm{nm}, 247 \mathrm{~nm}$ 吸收峰减弱并消失, 同时, 在 $358 \mathrm{~nm}$ 产 生一个新的吸收峰; 而在可见光区, 归属为 $\mathrm{Fe}(\mathrm{II}) \rightarrow$ $\mathrm{Cp}-\mathrm{C} \equiv \mathrm{C}-\mathrm{Ph}-\left(p-\mathrm{NMe}_{2}\right)$ 的 MLCT 带增强并发生 红移 (由 $440 \rightarrow 446 \mathrm{~nm}$ ). 在 272 及 $353 \mathrm{~nm}$ 处的两个 等吸收点表明该过程中存在质子化产物 ${ }^{[24]}$. 当滴加 $\mathrm{HBF}_{4} \cdot \mathrm{Et}_{2} \mathrm{O}$ 浓度至 10 倍当量时, 峰形不再变化, 表 明 $3 b$ 质子化为 $3 b \mathrm{H}^{+}$.

这种质子化过程中取代基推位电子效应的变化 同样导致3b电化学性质的变化(见图6). 随着 $\mathrm{HBF}_{4}$. $\mathrm{Et}_{2} \mathrm{O}$ 浓度的增加, $\mathrm{CV}$ 变化曲线中 $3 \mathbf{b}$ 中第一对二茂 铁的可逆氧化还原峰 $\mathrm{Fc}^{+} / \mathrm{Fc}$ 电位向正方向移动 $\left(E_{1 / 2}\right.$ 由 0.161 增大至 $0.231 \mathrm{~V}$ ); 同时, 由于 $\mathrm{N}$ 原子孤对电 子对与质子络合, 其在 $0.632 \mathrm{~V}$ 处归属为一 $\mathrm{NMe}_{2}^{+}$/ $-\mathrm{NMe}_{2}$ 氧化峰发生正移, 直至消失. 这个结果在 $\mathbf{3 b}$

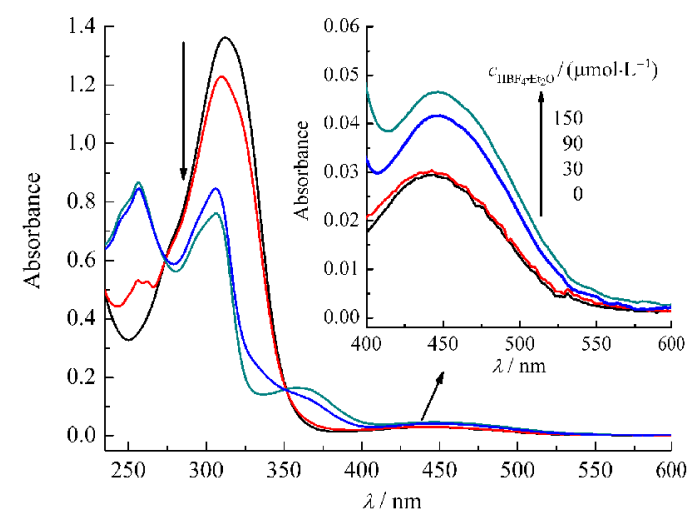

图 $53 \mathrm{~b}\left(30 \mu \mathrm{mol} \cdot \mathrm{L}^{-1}\right)$ 在乙腈中谪定 $\mathrm{HBF}_{4} \cdot \mathrm{Et}_{2} \mathrm{O}$ (0-150 $\left.\mu \mathrm{mol} \cdot \mathrm{L}^{-1}\right)$ 的紫外-可见吸收谱图

Fig.5 UV-Vis spectra of $3 b\left(30 \mu \mathrm{mol} \cdot \mathrm{L}^{-1}\right)$ on titration with $\mathrm{HBF}_{4} \cdot \mathrm{Et}_{2} \mathrm{O}\left(0-150 \mu \mathrm{mol} \cdot \mathrm{L}^{-1}\right)$ in $\mathrm{CH}_{3} \mathrm{CN}$ solution inset: enlarge figure in the range of $400-600 \mathrm{~nm}$ 

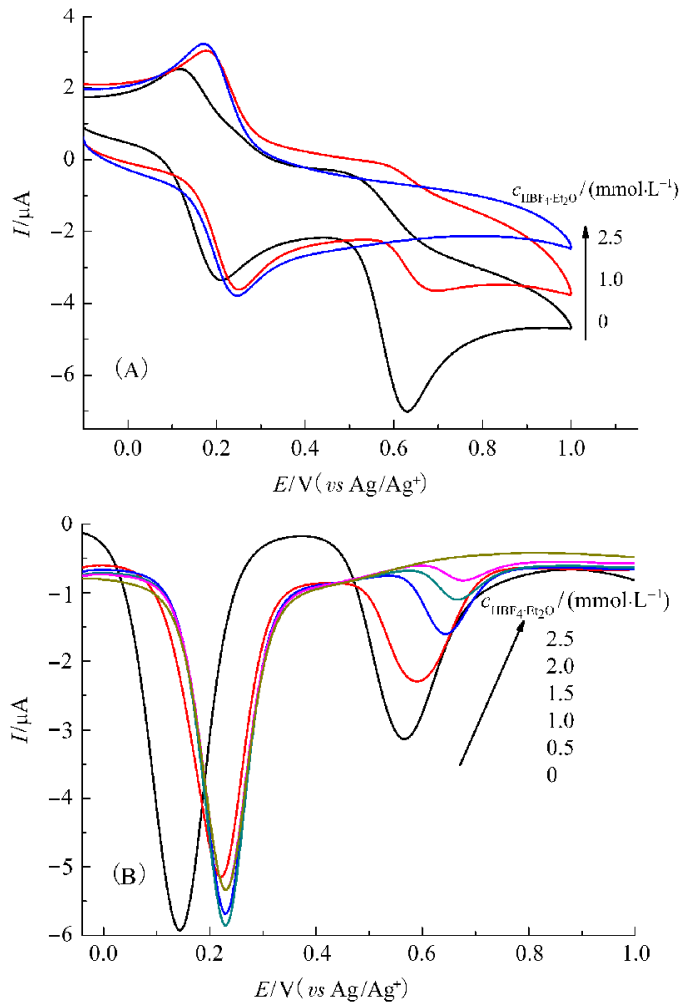

图 $63 \mathrm{~b}\left(500 \mu \mathrm{mol} \cdot \mathrm{L}^{-1}\right)$ 在乙腈中滴定 $\mathrm{HBF}_{4} \cdot \mathrm{Et}_{2} \mathrm{O}$

$\left(0-2.5 \mathrm{mmol} \cdot \mathrm{L}^{-1}\right)$ 的循环伏安 $(A)$ 和

常规差分脉冲伏安谱图(B)

Fig.6 CVs (A) and DPVs (B) of $3 \mathrm{~b}\left(500 \mu \mathrm{mol} \cdot \mathrm{L}^{-1}\right)$

on titration with $\mathrm{HBF}_{4} \cdot \mathrm{Et}_{2} \mathrm{O}\left(0-2.5 \mathrm{mmol} \cdot \mathrm{L}^{-1}\right)$ in $\mathrm{CH}_{3} \mathrm{CN}$ solution

加酸的 DPV 变化曲线中得到了进一步的印证, 随着 酸的浓度加大, 二茂铁的半波电位 $E_{\mathrm{p}}$ 不断增大 $\left(\Delta E_{\mathrm{p}}=0.086 \mathrm{~V}\right)$, 而一 $\mathrm{NMe}_{2}^{+} /-\mathrm{NMe}_{2}$ 氧化峰正移, 减 弱至逐渐消失.

虽然 3c 中的 $\mathrm{N}$ 原子同样含有孤对电子, 但加 人大量 $\mathrm{HBF}_{4} \cdot \mathrm{Et}_{2} \mathrm{O}$ 后其吸收光谱及电化学性质并 没有很大的变化. 这可能是因为三芳胺与 $N, N$-二甲 基-苯胺相比为更弱的碱, 文献报道它们的 $\mathrm{p} K_{\mathrm{a}}$ 值分 别为 5.15 及 $-5.0^{[26]}$.

\section{3 结 论}

合成了三种含电子给体乙炔基桥连二茂铁化合 物 3a-3c, 这些化合物相比于一些含吸电子基的二 元化合物的 MLCT 光谱出现了一定程度蓝移, 但其 氧化态在近红外区有更低能量的配体内部电荷转移 (ILCT) 吸收. 三化合物都有稳定的 $\mathrm{Fc}^{+} / \mathrm{Fc}$ 可逆氧化 还原电位, 且 $3 b$ 与 $3 c$ 还出现 $\mathrm{Ph}-\mathrm{NR}_{2}$ 的氧化电 位. 对于含一 $\mathrm{NMe}_{2}$ 取代基 $\mathbf{3 b}$, 发现对质子表现出光
及电化学敏感性, 对设计合成新的化学传感器有一 定的参考价值.

\section{References}

1 Gust, D.; Moore, T. A.; Moore, A. L. Acc. Chem. Res., 2001, 34: 40

2 Sauvage, J. P.; Collin, J. P.; Chambron, J. C.; Guillerez, S.; Coudret, C.; Balzani, V.; Barigelletti, F.; Cola, L. D.; Flamigni, L. Chem. Rev., 1994, 94: 993

3 Bella, S. D. Chem. Soc. Rev., 2001, 30: 355

4 Fery-Forgues, S.; Delavaux-Nicot, B. J. Photochem. Photobiol. A: Chem., 2000, 132: 137

5 Debroy, P.; Roy, S. Coord. Chem. Rev., 2007, 251: 203

6 Peris, E. Coord. Chem. Rev., 2004, 248: 279

7 Liao, Y.; Eichinger, B. E.; Firestone, K. A.; Haller, M.; Luo, J. D.; Kaminsky, W.; Benedict, J. B.; Reid, P. J.; Jen, A. K. Y.; Dalton, L. R.; Robinson, B. H. J. Am. Chem. Soc., 2005, 127: 2758

8 Delavaux-Nicot, B.; Maynadie, J.; Lavabre, D.; Fery-Forgues, S. Inorg. Chem., 2006, 45: 5691

9 Guldi, D. M.; Maggini, M.; Scorrano, G.; Prato, M. J. Am. Chem. Soc., 1997, 119: 974

10 Ceccon, A.; Santi, S.; Orian, L.; Bisello, A. Coord. Chem. Rev., 2004, 248: 683

11 Cuffe, L.; Hudson, R. D. A.; Gallagher, J. F.; Jennings, S.; McAdam, C. J.; Connelly, R. B. T.; Manning, A. R.; Robinson, B. H.; Simpson, J. Organometallics, 2005, 24: 2051

12 Chawdhury, N.; Long, N. J.; Mahon, M. F.; Ooi, L.; Raithby, P. R.; Rooke, S.; White, A. J. P.; Williams, D. J.; Younus, M. J. Organomet. Chem., 2004, 689: 840

13 Chen, Y. J.; Pan, D. S.; Chiu, C. F.; Su, J. X.; Lin, S. J.; Kwan, K. S. Inorg. Chem., 2000, 39: 953

14 Polin, J.; Schottenberger, H. Org. Synth., 1998, 9: 411

15 Xiao, H. B.; Shen, H.; Lin, Y. G.; Su, J. H.; Tian, H. Dyes Pigments, 2007, 73: 224

16 Perrin, D. D.; Armarego, W. L. F.; Perrin, D. R. Purification of laboratory chemicals. 2th ed. Oxford: Pergamon Press, 1980

17 Muthiah, C.; Kumar, K. P.; Mani, C. A.; Swamy, K. K. C. J. Org. Chem., 2000, 65: 3733

18 Kocher, S.; Lang, H. J. Organomet. Chem., 2001, 637-639: 198

19 Barlow, S.; Marder, S. R. Chem. Commun., 2000: 1555

20 Ju, H. D.; Tao, X. T.; Wan, Y.; Shi, J. H.; Yang J. X.; Xin, Q.; Zou, D. C.; Jiang, M. H. Chem. Phys. Lett., 2006, 432: 321

21 Barlow, S.; O'ahare, D. Chem. Rev., 1997, 637-669: 97

22 Martınez, R.; Ratera, I.; Tarraga, A.; Molina, P.; Veciana, J. Chem. Commun., 2006: 3809

23 McGale, E. M.; Robinson, B. H.; Simpson, J. Organometallics, 2003, 22: 931

24 Zucchero, A. J.; Wilson, J. N.; Bunz, U. H. F. J. Am. Chem. Soc., 2006, 128: 11872

25 García-Acosta, B.; Martínez-Mánez, R.; Sancenon, F.; Soto, J.; Rurack, K.; Spieles, M.; García-Breijo, E.; Gil, L. Inorg. Chem., 2007, 46: 3123

26 Goodall, W.; Williams, J. A. G. Chem. Commun., 2001: 2514 